\title{
THE LORENZ ATTRACTOR IS MIXING
}

\author{
STEFANO LUZZATTO, IAN MELBOURNE, AND FREDERIC PACCAUT
}

\begin{abstract}
We study a class of geometric Lorenz flows, introduced independently by Afrămovič, Bykov \& Sil'nikov and by Guckenheimer \& Williams, and give a verifiable condition for such flows to be mixing. As a consequence, we show that the classical Lorenz attractor is mixing.
\end{abstract}

\section{InTRODUCTION AND STATEMENT OF RESULTS}

1.1. The Lorenz equations. Many systems of nonlinear differential equations that were first studied almost 50 years ago and which were motivated mainly by problems in geophysical and astrophysical fluid dynamics and dynamical meteorology [2, 8, 15, 22, remain difficult to understand rigorously to the present day. In 1963, Lorenz [17] introduced the following system of differential equations:

$$
\begin{aligned}
& \dot{x}=10(y-x) \\
& \dot{y}=28 x-y-x z \\
& \dot{z}=x y-\frac{8}{3} z
\end{aligned}
$$

Approximate numerical studies of these equations led Lorenz to emphasise the possibility and importance of sensitive dependence on initial conditions even in such simplified models of natural phenomena. A combination of results obtained over the last 25 years 1, 14, 19, [24,29] and culminating in the work of Tucker [26, 27] gives the following statement (see [25, 28, for detailed surveys):

The Lorenz equations admit a robust attractor A which supports a "physical" ergodic invariant probability measure $\nu$ with a positive Lyapunov exponent

Recall that the measure $\nu$ is called physical, or Sinai-Bowen-Ruelle (SRB), if for Lebesgue almost every solution $u(t) \in \mathbb{R}^{3}$ starting close to $A$ and all continuous functions $h: \mathbb{R}^{3} \rightarrow \mathbb{R}$,

$$
\lim _{T \rightarrow \infty} \frac{1}{T} \int_{0}^{T} h(u(t)) d t=\int_{A} h d \nu .
$$

In this paper we take a further step in the understanding of the statistical properties of the Lorenz attractor. A measure $\nu$ is mixing for a flow $\Phi_{t}$ if

$$
\nu\left(\Phi_{t}(A) \cap B\right) \rightarrow \nu(A) \nu(B)
$$

for all measurable sets $A, B$, as $t \rightarrow \infty$. We say that the Lorenz attractor is mixing if the $\mathrm{SRB}$ measure $\nu$ mentioned above is mixing. We prove the following

Theorem 1. The Lorenz attractor is mixing. 
In fact, our result shows that the Lorenz attractor is stably mixing: sufficiently small $C^{1}$ perturbations of the flow are mixing.

There are relatively few explicit examples of flows that have been proved to be mixing. Anosov 4] showed that geodesic flows for compact manifolds of negative curvature are mixing, and this was generalised [16] to include contact flows. Moreover, mixing persists under $C^{1}$ perturbations. For codimension one Anosov flows [20] and for Anosov flows with a global infranil cross-section [5], the set of mixing flows is $C^{1}$ open, but the corresponding result for general Anosov flows is not known. Recently [11] it was shown that mixing holds for a $C^{1}$-open and $C^{r}$-dense set of $C^{r}$ Anosov flows for all $r \geq 1$. (In these references, mixing is proved for any equilibrium measure for a Hölder potential.) However, the conditions for stable mixing in [10, 11] for Anosov and uniformly hyperbolic (Axiom A flows) are not easily verifiable.

The Lorenz attractor is an example of a singular hyperbolic attractor [18 (uniformly hyperbolic, except for a singularity due to the attractor containing an equilibrium). Somewhat surprisingly, we show that the singular nature of the Lorenz attractor assists in the search for a verifiable condition for mixing.

1.2. Geometric Lorenz attractors. Afră̌movič, Bykov and Sil'nikov [1] and Guckenheimer and Williams [14,29] introduced a geometric model that is an abstraction of the numerically-observed features possessed by solutions to (1.1). Tucker [26, 27] proved that the geometric model is valid, so the Lorenz equations define a geometric Lorenz flow.

Accordingly, our approach to Theorem 1 is to establish mixing for geometric Lorenz flows satisfying certain hypotheses, and then to verify from [26, 27] that the hypotheses are satisfied for the Lorenz equations. Roughly speaking, a geometric Lorenz flow is the natural extension of a geometric Lorenz semiflow which is itself a suspension flow built over a certain type of one-dimensional expanding map $f$. Precise definitions are given in Section [2, In the literature, a standard assumption is that the map $f$ is locally eventually onto (l.e.o.), see Definition 3. We prove that this is a sufficient condition for the corresponding flow to be mixing.

Theorem 2. Let $f_{t}$ be a geometric Lorenz flow. Suppose that the associated one-dimensional map $f$ is l.e.o. Then $f_{t}$ is mixing (and even Bernoulli).

The remainder of the paper is structured as follows. In Section 2, we discuss the geometric Lorenz flow and its relations to the Lorenz attractor. In particular, we indicate how Theorem 1 follows from Theorem 2. In Section [3, we prove Theorem 2, In Section 4, we discuss extensions of our main results and some related future directions.

\section{The Lorenz ATtractor is A GeOMEtric Lorenz Flow}

In this section we collect and organise several results from the existing literature on the relation between the Lorenz attractor and geometric Lorenz flows. We recall first of all some basic relevant facts about the Lorenz equations (1.1), see [25]. The origin is an equilibrium of saddle type with two negative (stable) and one positive (unstable) eigenvalues $\lambda_{s s}<\lambda_{s}<0<\lambda_{u}$. It is also the case that $\lambda_{u}>\left|\lambda_{s}\right|$. 
Suppose that a finite number of nonresonance conditions are satisfied so that the vector field is smoothly linearisable in a neighbourhood of 0 . In these coordinates, the flow near 0 has the form $\left(x_{1}, x_{2}, x_{3}\right) \mapsto\left(e^{\lambda_{u} t} x_{1}, e^{\lambda_{s s} t} x_{2}, e^{\lambda_{s} t} x_{3}\right)$. By a linear rescaling, we can suppose that the domain of linearisation of the flow includes the cube $[-1,1]^{3}$. Define $\Sigma=\left\{\left(x_{1}, x_{2}, x_{3}\right):\left|x_{1}\right|,\left|x_{2}\right| \leq 1, x_{3}=1\right\}$ and $\widetilde{\Sigma}=\left\{\left(x_{1}, x_{2}, x_{3}\right): x_{1}= \pm 1,\left|x_{2}\right|,\left|x_{3}\right| \leq 1\right\}$. Then we define the first hit map $P_{0}: \Sigma \rightarrow \widetilde{\Sigma}$ by

$$
P_{0}\left(x_{1}, x_{2}, 1\right)=\left(e^{\lambda_{u} r_{0}} x_{1}, e^{\lambda_{s s} r_{0}} x_{2}, e^{\lambda_{s} r_{0}}\right)=\left(\operatorname{sgn} x_{1}, \widetilde{x}_{2}, \widetilde{x}_{3}\right),
$$

where $r_{0}$ is the "time of flight" from $\Sigma$ to $\widetilde{\Sigma}$. Solving $e^{\lambda_{u} r_{0}} x_{1}=\operatorname{sgn} x_{1}$ for $r_{0}=-\left(\ln \left|x_{1}\right|\right) / \lambda_{u}$, we obtain

$$
P_{0}\left(x_{1}, x_{2}, 1\right)=\left(\operatorname{sgn} x_{1},\left|x_{1}\right|^{\beta} x_{2},\left|x_{1}\right|^{\alpha}\right),
$$

where $\alpha=\left|\lambda_{s}\right| / \lambda_{u} \in(0,1)$ and $\beta=\left|\lambda_{s s}\right| / \lambda_{u}>0$. Note that $P_{0}: \Sigma \rightarrow \widetilde{\Sigma}$ is well-defined on $\Sigma \backslash W^{s}(0)$ and that $r_{0}$ has a logarithmic singularity at $x_{1}=0$.

Tucker [26, 27] proves that there exists a compact trapping region $N \subset \Sigma$ such that the Poincaré first return map $P: N \backslash W^{s}(0) \rightarrow N$ is well defined. We can decompose $P=P_{1} \circ P_{0}$ where $P_{1}$ is the first-hit map between $\widetilde{\Sigma}$ and $\Sigma$. Note that $P_{1}$ is a diffeomorphism where it is defined, and that the time of flight $r_{1}$ for $P_{1}$ is bounded. Hence the time of flight $r=r_{0}+r_{1}$ for the full return map $P$ is smooth except for a logarithmic singularity at $x_{1}=0$. Moreover, the following crucial hyperbolicity estimate holds.

Lemma 2.1 (27]). The return map $P$ admits a forward invariant cone field. In other words, there exists a cone $\mathcal{C}(u)$ inside $\Sigma$ at each point $u \in N \backslash W^{s}(0)$ such that $(d P)_{u} \mathcal{C}(u)$ is strictly contained inside $\mathcal{C}(P u)$.

Moreover, there exist constants $c>0, \tau>1$, such that for each $u \in N \backslash W^{s}(0)$,

$$
\left\|\left(d P^{n}\right)_{u} v\right\| \geq c \tau^{n}\|v\|
$$

for every $v \in \mathcal{C}(u)$ and $n \geq 1$.

A consequence of Lemma 2.1] is that the return map $P$ has an invariant stable foliation with $C^{1+\varepsilon}$ leaves for some $\varepsilon>0$. Let $I=[-1,1]$. We obtain a singular one-dimensional map $f: I \rightarrow I$ by quotienting along stable leaves. At the same time, $r$ reduces to a singular $\operatorname{map} r: I \rightarrow \mathbb{R}^{+}$. Let $J=I \backslash\{0\}$. Then it is immediate that on $J$ :

$\mathbf{f}(\mathbf{i}): f$ is $C^{1+\varepsilon}$.

f(ii): $\left|f^{(n)}\right| \geq c \tau^{n}$ for all $n \geq 1$.

f(iii): $C^{-1}|x|^{\alpha-1} \leq f^{\prime}(x) \leq C|x|^{\alpha-1}$,

$\mathbf{r}(\mathbf{i}): r(x) \rightarrow \infty$ as $x \rightarrow 0$,

$\mathbf{r}(\mathrm{ii}):|r(x)-r(y)| \leq C|\ln | x|-\ln | y||$ for all $x, y>0$ and all $x, y<0$,

where $c>0, \tau>1$ are the constants in Lemma 2.1, $\alpha=\left|\lambda_{s}\right| / \lambda_{u} \in(0,1)$, and $C \geq 1$ is a constant. Note that it follows from $\mathbf{r}$ (ii) that $r$ is Lebesgue integrable and is $C^{1}$ on $J$.

We have described how to pass from the original three-dimensional flow defined by the Lorenz equations (1.1) to a two-dimensional Poincaré map $P: \Sigma \rightarrow \Sigma$ and then to a one-dimensional expanding map $f: I \rightarrow I$. The process is reversible: taking the natural 
extension of $f$ recovers the Poincaré map $P$ and taking the suspension of the $P$ by the roof function $r$ recovers the original Lorenz flow. Alternatively, these steps can be carried out in the opposite order to recover the Lorenz flow as the natural extension of the suspension semiflow of the map $f$ by the roof function $r$. The latter viewpoint is the geometric Lorenz flow construction [14,29]. For completeness, the notions of suspension and natural extension are recalled at the end of this section.

Definition 1. Let $f: I \rightarrow I$. Assume that $f(0)$ is undefined, with $f\left(0^{+}\right)=-1$ and $f\left(0^{-}\right)=+1$. It is assumed that $f(1) \in(0,1)$ and $f(-1) \in(-1,0)$. (See Figure 11) If conditions $\mathbf{f}(\mathbf{i})-\mathbf{f}(\mathbf{i i i})$ are satisfied, then $f$ is a Lorenz-like expanding map.

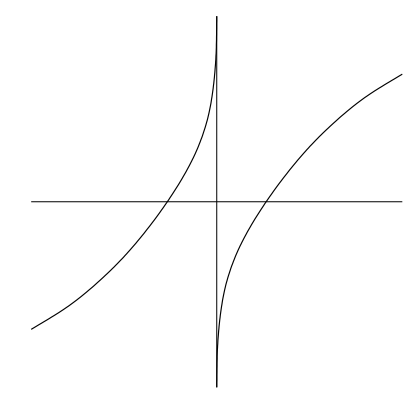

Figure 1. The graph of a Lorenz-like expanding map $f:[-1,1] \rightarrow[-1,1]$.

Definition 2. A semiflow $f_{t}$ is called a geometric Lorenz semiflow if it is the suspension over a Lorenz-like expanding map $f: I \rightarrow I$ by a roof function $r$ satisfying conditions $\mathbf{r}(\mathbf{i})$ and $\mathbf{r}(\mathbf{i i})$. A flow $f_{t}$ is called a geometric Lorenz flow if it is the natural extension of a geometric Lorenz semiflow.

The results of Tucker described above imply that the Lorenz equations indeed define a geometric Lorenz flow. Moreover, as outlined below, Tucker showed that the associated expanding map $f: I \rightarrow I$ is transitive for the Lorenz equations, thus establishing the existence of the Lorenz attractor.

In the following definition, we stress that $f(0)$ is undefined. In particular, the endpoints \pm 1 have no preimages.

Definition 3. The map $f: I \rightarrow I$ is locally eventually onto (l.e.o.) if for any open set $U \subset J$, there exists $k \geq 0$ such that $f^{k} U$ contains $(0,1)$.

Remark 2.2. Since $f(0,1)$ contains $(-1,0)$ and vice versa, we could equally use $(-1,0)$ instead of $(0,1)$ in the definition of l.e.o. Clearly, the l.e.o. property implies that $f$ is topologically transitive. The exact formulation of l.e.o. varies in the literature, but our definition agrees with the one in [12].

Remark 2.3. It was anticipated in 29 that $\mathbf{f}(\mathbf{i i})$ would hold with $c=1, \tau>\sqrt{2}$. It is easy to check that this is a sufficient condition for $f$ to be l.e.o.

Surprisingly [27, for the actual Lorenz equations it turns out that $f^{\prime}(x)<1$ for certain values of $x \in I$. Nevertheless, it is still the case that $f$ is l.e.o., see Tucker [27]. 
It can be shown that Lorenz-like expanding maps satisfying the l.e.o. condition have a unique ergodic probability measure $\mu$ that is equivalent to Lebesgue (see for example Section 31). The suspended measure $\nu=\mu^{r}$ defines an SRB measure for the geometric Lorenz flow. In particular, Theorem 1 follows from Theorem 2 . The latter result is proved in Section 3 .

Remark 2.4. The approach in this paper establishes weak mixing for geometric Lorenz semiflows. It then follows from Ratner [21] that such semiflows, and the corresponding flows, are Bernoulli. In particular, they are mixing.

Suspensions and natural extensions. As promised, we end this section by recalling the notions of suspension and natural extension. Let $(X, \mu)$ be a probability space and $f: X \rightarrow X$ a (noninvertible) measure preserving transformation. Let $r: X \rightarrow \mathbb{R}^{+}$be an $L^{1}$ roof function. Define the suspension $X^{r}=\{(x, u): x \in X, u \in[0, r(x)]\} / \sim$ where $(x, r(x)) \sim$ $(f x, 0)$. Form the suspension semiflow $f_{t}: X^{r} \rightarrow X^{r}$ given by $f_{t}(x, u)=(x, u+t)$ computed modulo identifications. An invariant probability measure is given by $\mu^{r}=\mu \times \ell / \int r d m$ where $\ell$ is Lebesgue measure.

Next suppose that $f_{t}: \Omega \rightarrow \Omega$ is a semiflow preserving a probability measure $\nu$. Form the natural extension (or inverse limit) $\widehat{\Omega}$ consisting of curves $\{x(s)\}_{s \geq 0}$ in $\Omega$ satisfying $f_{t}(x(s))=x(s-t)$ for all $s \geq t \geq 0$. An invertible flow $\widehat{f}_{t}: \widehat{\Omega} \rightarrow \widehat{\Omega}$ is given by $\widehat{f}_{t}\{x(s)\}_{s \geq 0}=$ $\{x(s+t)\}_{s \geq 0}$. The projection $\pi: \widehat{\Omega} \rightarrow \Omega,\{x(s)\}_{s \geq 0} \mapsto x(0)$ defines a semiconjugacy between $\widehat{f}_{t}$ and $f_{t}$ and there is a unique $\widehat{f}_{t}$-invariant measure $\nu$ on $\widehat{\Omega}$ such that $\pi$ is measure preserving.

\section{MiXing FOR GEOMETRIC LORENZ FLOWS}

In this section, we prove Theorem 2. Namely, we show that if $f$ is an l.e.o. expanding map of Lorenz-type and $r$ is a logarithmic roof function, then the corresponding geometric Lorenz flow is mixing. By Remark 2.4 it is enough to prove that the corresponding geometric Lorenz semiflow is weak mixing.

Let $f: I \rightarrow I$ be a Lorenz-like expanding map. As shown in Diaz-Ordaz [9], $f$ admits an induced map $F$ that is Gibbs-Markov. More precisely there is an open interval $Y \subset I$ containing 0 and (modulo a set of Lebesgue measure zero) there is a partition $\mathcal{P}=\{\omega\}$ of $Y$ consisting of intervals, and a return function $R: Y \rightarrow \mathbb{N}$ constant on partition elements such that the induced map $F(x)=f^{R(x)}(x)$ restricts to a diffeomorphism $\left.F\right|_{\omega}: \omega \rightarrow Y$ for each partition element $\omega$, and such that the following conditions are satisfied:

(a) There exists $\lambda>1$ such that $\left|D F_{\omega}\right| \geq \lambda$ for each $\omega$.

(b) For all $\omega, \log g_{\omega}$ is Hölder (uniformly in $\omega$ ), where $g$ is the Jacobian of the inverse of $\left.F\right|_{\omega}: \omega \rightarrow Y$.

(c) $R$ is Lebesgue integrable.

A standard Folklore Theorem in dynamics says that conditions (a)-(c) implies the existence of a unique ergodic invariant measure $\mu$ for $f: I \rightarrow I$ that is absolutely continuous with respect to Lebesgue and whose support includes $Y$. In addition, the construction of 9 ] satisfies 
(d) $0 \notin \overline{f^{k} \omega}$ for $0 \leq k<R(\omega)$ for each $\omega$.

The following Livšic regularity result is due to Bruin et al. [6].

Lemma 3.1. Suppose that $f: I \rightarrow I$ admits an induced map $F: Y \rightarrow Y$ satisfying conditions (a)-(d) above, with absolutely continuous ergodic measure $\mu$. Let $r: I \rightarrow \mathbb{R}^{+}$be a Hölder roof function with a logarithmic singularity at 0 . Let $\psi: I \rightarrow S^{1}$ be a $\mu$-measurable function satisfying

$$
e^{i r}=(\psi \circ T) \psi^{-1}, \text { a.e. }
$$

Then $\psi$ has a version that is Hölder on $Y$.

Sketch proof. We provide the details required for the reader to pass between the formulation in [6] and the statement of the lemma. By conditions (a)-(c), the map $f: I \rightarrow I$ can be modelled by a Young tower 31] with base $Y$. The appropriate measure for the tower has a Hölder density, so by condition (d), Bruin et al. [6, Theorem 2] applies to guarantee that $\psi$ is Hölder on any fixed partition element of $Y$ and hence on the whole of $Y$ ([․ Remark 3]). The tower metric in [6] coincides with the Euclidean metric when restricted to $Y$, so $\psi$ is Hölder in the original metric.

Remark 3.2. Related Livšic regularity results can be found in [13. The arguments in [6, 13. are different from each other leading to distinct results, and the approach in [6] turns out to more convenient for our purposes.

Now let $r: I \rightarrow \mathbb{R}^{+}$be a Hölder roof function with a logarithmic singularity at 0 , and let $f_{t}$ be the corresponding geometric Lorenz semiflow with ergodic measure $\mu^{r}$.

Lemma 3.3. Suppose that the geometric Lorenz semiflow is not weak mixing. Then there exists a constant $a>0$ and a measurable eigenfunction $\psi: X \rightarrow S^{1}$ continuous on $\bigcup_{k \geq 0} f^{k} Y$, such that

$$
e^{i a r}=(\psi \circ f) \psi^{-1} \text { a.e. }
$$

Proof. It can be taken as the definition of weak mixing that $\phi \circ f_{t}=e^{i a t} \phi$ has no measurable solutions $\phi: X^{r} \rightarrow S^{1}$ for $a>0$. Suppose that $\phi$ is such a measurable solution. It follows from Fubini that there exists $\epsilon>0$ with $r>\epsilon$ a.e. such that $\phi \circ f_{t}(x, \epsilon)=e^{i a t} \phi(x, \epsilon)$ for almost every $x \in X$. Set $t=r(x)$ and $\psi(x)=\phi(x, \epsilon)$. Since $f_{r(x)}(x, \epsilon)=(f x, \epsilon)$ we obtain that $\psi \circ f=e^{i a r} \psi$. Thus $\psi$ is a measurable solution to equation (3.1). By Lemma 3.1. there is a solution $\psi$ that is Hölder continuous on $Y$.

It is now straightforward to show that $\psi$ is continuous on $\bigcup_{k \geq 0} f^{k} Y$. Suppose that $z=f^{k} y$ where $y \in Y$. Since $f(0)$ is undefined, it is certainly the case that $f^{j} y \neq 0$ for $j=0, \ldots, k-1$. Hence we can choose an open set $U \subset Y$ containing $y$ such that $0 \notin f^{j} U$ for $0 \leq j \leq k-1$. In particular, there exists $\gamma \in(0,1)$ such that $\psi$ is $C^{\gamma}$ on $Y$ and at the same time $e^{i a r_{k}}$ is $C^{1}$ on $U$.

Let $z_{i}=f^{k} y_{i}$ where $y_{i} \in U$ for $i=1$,2. Applying equation (3.1) in the form $\psi \circ f^{k}=$ $e^{i a r_{k}} \psi$, we obtain

$$
\psi\left(z_{1}\right) \psi\left(z_{2}\right)^{-1}=\psi\left(y_{1}\right) \psi\left(y_{2}\right)^{-1} e^{i a r_{k}\left(y_{1}\right)} e^{-i a r_{k}\left(y_{2}\right)}
$$


so by $\mathbf{f}(\mathbf{i i})$,

$$
\left|\psi\left(z_{1}\right) \psi\left(z_{2}\right)^{-1}\right| \leq D\left|y_{1}-y_{2}\right|^{\gamma} \leq D\left(c \lambda^{k}\right)^{-\gamma}\left|z_{1}-z_{2}\right|^{\gamma} .
$$

Hence $\psi$ is Hölder on $f^{k} U$ and so is certainly continuous at $z$ as required.

Proof of Theorem 2. Suppose that $f_{t}$ is not weak mixing. By Lemma 3.3, there exists $a>0$ and a measurable eigenfunction $\psi: I \rightarrow S^{1}$ satisfying (3.1), such that $\psi$ is continuous on $\bigcup_{k \geq 0} f^{k} Y$. Since $f$ is l.e.o., $\psi$ is continuous on $(-1,1)$. In particular, $\psi$ is continuous at $f( \pm 1)$.

Iterate equation (3.1) to obtain

$$
e^{i a r} e^{i a r \circ f}=\left(\psi \circ f^{2}\right) \psi^{-1} .
$$

We evaluate this equation along a sequence $x_{n}>0$ with $x_{n} \rightarrow 0$. We have the limits $\psi\left(x_{n}\right) \rightarrow \psi(0), \psi\left(f^{2} x_{n}\right) \rightarrow \psi(f(-1))$, and $r\left(f x_{n}\right) \rightarrow r(-1)$. Hence, the right-hand-side of equation (3.2) and the second factor on the left-hand-side converge as $n \rightarrow \infty$. To obtain a contradiction, it suffices to choose $x_{n} \rightarrow 0$ so that $e^{i a r\left(x_{n}\right)}$ does not converge. For $n$ sufficiently large, choose $b_{n}>r\left(\epsilon / 2^{n}\right)$ such that $e^{i a b_{n}}=(-1)^{n}$. Since $r$ is continuous on $I-\{0\}$ and $r(x) \rightarrow \infty$ as $x \rightarrow 0$, there exists $x_{n}>0$ such that $r\left(x_{n}\right)=b_{n}$. As required, $x_{n} \rightarrow 0$ and $e^{i a r\left(x_{n}\right)}$ does not converge.

\section{Extensions AND FUture DiRECTIONS}

In this paper, we have focused on geometric Lorenz attractors satisfying a locally eventually onto condition. In this section, we discuss how to relax the l.e.o. condition, and we describe how our results extend to larger classes of singular attractors. We end by mentioning some future directions.

The l.e.o. assumption occurs in much of the literature since it is a verifiable property of the flow that guarantees topological transitivity of $f$. Moreover, it holds for the Lorenz equations (1.1) themselves [27]. However, topological transitivity for $f: I \rightarrow I$ is not crucial in this paper. We could simply work with the unique ergodic component whose support includes the set $Y$ introduced in Section 3. Passing to the natural extension of the suspension over this component yields a geometric Lorenz attractor with an SRB measure as before.

The proof of mixing for this attractor also relies to some extent on the l.e.o. property, but it is clear that the proof goes through under a much weaker condition: namely that a preimage of a forward image of 1 or -1 lies in $Y$. (That is, there exist integers $k, \ell \geq 0$ such that $f^{\ell}(1) \in f^{k} Y$ or $f^{\ell}(-1) \in f^{k} Y$.) This condition is open and dense within the class of Lorenz-like expanding maps, and in principle, it can be verified by a finite computation.

Next, we note that the specific structure of Lorenz-like expanding maps is not so crucial for the proof of mixing. Properties $\mathbf{f}(\mathbf{i})-\mathbf{f}(\mathbf{i i i})$ could be relaxed, or altered completely, provided $f$ admits an induced map $F: Y \rightarrow Y$ satisfying conditions (a)-(d). The existence of a Gibbs-Markov induced map is a very general condition, see [3, 7]. It clearly holds for uniformly expanding maps and, we have made use of the the recent work of Diaz-Ordaz 9 for Lorenz-like expanding maps. 
Condition $\mathbf{f}(\mathbf{i i})$ in the definition of the Lorenz-like expanding map corresponds to the eigenvalue condition $\lambda_{u}>\left|\lambda_{s}\right|$ which is valid for the Lorenz attractor. The resulting class of geometric Lorenz attractors are sometimes called expanding. There are also contracting geometric Lorenz attractors [23] that arise when $\lambda_{u}<\left|\lambda_{s}\right|$. It seems likely that such attractors can be shown to be mixing using the ideas in this paper.

In situations where Lemma 2.1fails, it may not be possible to reduce to a one-dimensional expanding map. However, it is plausible that the Poincaré map $P$ could be modelled by an "unquotiented" Young tower as in [30] to which the ideas in this paper might still be applicable.

In this paper, we have established mixing. An interesting open question is to prove results on the speed of mixing. In a different direction, it would be interesting to derive statistical limit laws such as the central limit theorem and invariance principles for the Lorenz attractor.

Acknowledgements. This research was supported in part by EPSRC Grant GR/S11862/01. IM is greatly indebted to the University of Houston for the use of e-mail, given that pine is currently not supported on the University of Surrey network.

\section{REFERENCES}

[1] V. S. Afraĭmovič, V. V. Bykov, and L. P. Sil'nikov, The origin and structure of the Lorenz attractor, Dokl. Akad. Nauk SSSR 234 (1977), 336-339.

[2] D. W. Allan, On the behaviour of systems of coupled dynamos, Proc. Cambridge Philos. Soc. 58 (1962).

[3] José F. Alves, Stefano Luzzatto, and Vilton Pinheiro, Lyapunov exponents and rates of mixing for one-dimensional maps., Ergodic Th. \& Dyn. Syst. 24 (2004), 637-657.

[4] D. V. Anosov, Geodesic flows on closed Riemann manifolds with negative curvature., Proceedings of the Steklov Institute of Mathematics, No. 90 (1967). Translated from the Russian by S. Feder, American Mathematical Society, Providence, R.I., 1969.

[5] Rufus Bowen, Mixing Anosov flows, Topology 15 (1976), 77-79.

[6] Henk Bruin, Mark Holland, and Matthew Nicol, Livsic regularity for Markov systems (2004) (Preprint).

[7] Henk Bruin, Stefano Luzzatto, and Sebastian van Strien, Decay of correlations in one-dimensional dynamics, Ann. Sci. Éc. Norm. Sup. 36 (2003), 621-646.

[8] E.C. Bullard, The stability of a homopolar dynamo, Cambridge Phil. Soc 51 (1955), 744-760.

[9] Karla Diaz-Ordaz, Decay of correlations for non-Hölder observables for expanding Lorenz-like onedimensional maps (2004) (In preparation).

[10] M Field, I Melbourne, and A Török, Stability of mixing for hyperbolic flows (May 2003) (Preprint).

[11] _ Stability of rapid mixing for hyperbolic flows (July 2004) (Preprint).

[12] Paul Glendinning and Colin Sparrow, Prime and renormalisable kneading invariants and the dynamics of expanding Lorenz maps, Phys. D 62 (1993), 22-50, Homoclinic chaos (Brussels, 1991).

[13] Sébastien Gouëzel, Regularity of coboundaries for non uniformly expanding Markov maps (2004) (Preprint).

[14] John Guckenheimer and R. F. Williams, Structural stability of Lorenz attractors, Inst. Hautes Études Sci. Publ. Math. (1979), 59-72.

[15] Raymond Hide, Geomagnetism, 'vacillation', atmospheric predictability and deterministic chaos (2004) (Contribution to a Symposium on 'Paths of Discovery'). 
[16] Anatole Katok, Infinitesimal Lyapunov functions, invariant cone families and stochastic properties of smooth dynamical systems, Ergodic Theory Dynam. Systems 14 (1994), 757-785, With the collaboration of Keith Burns.

[17] E. D. Lorenz, Deterministic nonperiodic flow, J. Atmosph. Sci. 20 (1963), 130-141.

[18] C. A. Morales, M. J. Pacifico, and E. R. Pujals, Singular hyperbolic systems, Proc. Amer. Math. Soc. 127 (1999), 3393-3401.

[19] Ya. Pesin, Dynamical systems with generalized hyperbolic attractors: hyperbolic, ergodic and topological properties, Ergod. Th. \& Dynam. Sys. 12 (1992), 123-151.

[20] Joseph F. Plante, Anosov flows, Amer. J. Math. 94 (1972), 729-754.

[21] M. Ratner, Bernoulli flow over maps of the interval, Israel J. Math. 31 (1978), 298-314.

[22] Tsuneji Rikitake, Oscillations of a system of disk dynamos, Proc. Cambridge Philos. Soc. 54 (1958), $89-105$.

[23] Alvaro Rovella, The dynamics of perturbations of the contracting Lorenz attractor, Bol. Soc. Brasil. Mat. (N.S.) 24 (1993), 233-259.

[24] E. A. Sataev, Invariant measures for hyperbolic mappings with singularities, Uspekhi Mat. Nauk 47 (1992), 147-202, 240. (Russian)

[25] C. Sparrow, The Lorenz equations: bifurcations, chaos and strange attractors, Applied Mathematical Sciences, vol. 41, Springer Verlag, Berlin, 1982.

[26] Warwick Tucker, The Lorenz attractor exists, C. R. Acad. Sci. Paris Sér. I Math. 328 (1999), 11971202.

[27] _ A rigorous ODE solver and Smale's 14th problem, Found. Comput. Math. 2 (2002), 53-117.

[28] Marcelo Viana, Dynamical systems: moving into the next century, Mathematics unlimited-2001 and Beyond, Springer, Berlin, 2001, pp. 1167-1178.

[29] R. F. Williams, The structure of Lorenz attractors, Inst. Hautes Études Sci. Publ. Math. (1979), 7399.

[30] Lai-Sang Young, Statistical properties of dynamical systems with some hyperbolicity, Ann. of Math. (2) 147 (1998), 585-650.

[31] — Recurrence times and rates of mixing, Israel J. Math. 110 (1999), 153-188.

Mathematics Department, Imperial College, 180 Queen's Gate, London SW7 2AZ

E-mail address: stefano.luzzatto@imperial.ac.uk

$U R L$ : www.ma.ic.ac.uk/ luzzatto

Department of Mathematics and Statistics, School of ECM, University of Surrey GuildFord, Surrey GU2 7XH, UK

E-mail address: ism@login.math.uh.edu

$U R L$ : www.maths.surrey.ac.uk/showstaff?I.Melbourne

LAMFA UMR 6140 du CNRS, University of Picardie, 33, Rue Saint-Leu 80039 Amiens CEDEX 1, France

E-mail address: frederic.paccaut@u-picardie.fr

$U R L$ : lamfa.u-picardie.fr/paccaut/ 